\title{
Sarcopenia reduces quality of life in the long-term: longitudinal analyses from the English longitudinal study of ageing
}

\author{
Nicola Veronese $^{1} \cdot$ Ai Koyanagi $^{2,3} \cdot$ Emanuele Cereda $^{4} \cdot$ Stefania Maggi $^{5} \cdot$ Mario Barbagallo $^{1} \cdot$ Ligia J. Dominguez $^{1}$. \\ Lee Smith ${ }^{6}$
}

Received: 24 December 2021 / Accepted: 9 February 2022 / Published online: 25 February 2022

(c) The Author(s) 2022

\section{Key summary points}

Aim To examine the association between sarcopenia at baseline and changes in quality of life at 10 years follow-up in a large representative sample of older English adults.

Findings After considering numerous confounders, sarcopenia at baseline was associated with a higher incidence of poor quality of life. People having sarcopenia at baseline reported significantly lower values in CASP-19 after ten years of follow-up.

Message Sarcopenia is an important and independent risk factor for poor quality of life in older people.

\begin{abstract}
Purpose Mixed findings exist for sarcopenia/quality of life (QoL) relationship. Moreover, the majority of studies in this area have utilized a cross-sectional design or specific clinical populations. Therefore, the aim of the present study was to examine the association between sarcopenia at baseline and QoL at 10 years follow-up in a large representative sample of older English adults.

Methods Sarcopenia was diagnosed as having low handgrip strength and low skeletal muscle mass index. QoL was measured using the CASP (control, autonomy, self-realisation and pleasure)-19, with higher values reflecting higher QoL. Multivariable logistic regression analysis was conducted to assess prospective associations between sarcopenia at baseline and poor QoL at follow-up; generalized linear model with repeated measures was used for reporting mean changes during follow-up between sarcopenia and not.

Results Among 4044 older participants initially included at baseline (mean age: 70.7 years; $55.1 \%$ females), 376 had sarcopenia. In the multivariable analysis, after adjusting for several potential confounders, sarcopenia at baseline was associated with a higher incidence of poor QoL (odds ratio, $\mathrm{OR}=5.82$; 95\% confidence interval, CI 3.45-9.82). After matching for QoL values at baseline and adjusting for potential confounders, people with sarcopenia reported significantly lower values in CASP-19 (mean difference $=-3.94 ; 95 \% \mathrm{CI}-4.77$ to -3.10 ).

Conclusions In this large representative sample of older English adults, it was observed that sarcopenia at baseline was associated with worse scores of QoL at follow-up compared to those without sarcopenia at baseline. It may be prudent to target those with sarcopenia to improve QoL.
\end{abstract}

Keywords Quality of life · Sarcopenia $\cdot$ ELSA $\cdot$ Older adults $\cdot$ Epidemiology $\cdot$ Longitudinal $\cdot$ Ageing

Nicola Veronese

nicola.veronese@unipa.it

1 Geriatric Unit, Department of Internal Medicine and Geriatrics, University of Palermo, via del Vespro, 141, 90127 Palermo, Italy

2 Research and Development Unit, Parc Sanitari Sant Joan de Déu, CIBERSAM, Dr. Antoni Pujadas, 42, 08830 Sant Boi de Llobregat, Barcelona, Spain
3 ICREA, Pg, Lluis Companys 23, 08010 Barcelona, Spain

4 Clinical Nutrition and Dietetics Unit, Fondazione IRCCS Policlinico San Matteo, Pavia, Italy

5 Institute of Neuroscience, National Research Council, Padova, Italy

6 Centre for Health, Performance and Wellbeing, Anglia Ruskin University, Cambridge, UK 


\section{Background}

Quality of life (QoL) is one's perception of their position in life in the context of the culture and value systems in which one lives, and in relation to one's goals, expectations, standards and concerns [1]. QoL is indeed an important measure of overall health, including wellbeing [2], Health organizations emphasize that maintaining good QoL is of importance throughout the life course [2].

Importantly, QoL has been observed to be associated with mortality. For example, in one review of several studies, including approximately 1,200,000 participants, it was found that better QoL was associated with lower mortality risk [3]. This association has been found to be strong in older adults particularly when health-related QoL is used as an outcome. For example, in a prospective cohort study of 2373 persons, representative of the Spanish population aged 60 and older, it was found that changes in health-related QoL predicted mortality [4]. It is thus important to identify correlates of QoL in older adults to inform targeted interventions to improve QoL or maintain adequate levels. One potential but understudied correlate of QoL in older adults is that of sarcopenia.

Sarcopenia refers to "age-related muscle loss, affecting a combination of appendicular muscle mass, muscle strength, and/or physical performance measures" [5] and is now widely considered to be a disease [6]. Sarcopenia is plausibly linked to a reduction in QoL as it has been observed to be associated with higher rates of hospitalization, dependency, falls and disability [7]. In one systematic review, 11 studies investigating QoL in sarcopenic people were identified, and interestingly, the results were quite heterogenous showing either no difference in QoL between sarcopenic and non-sarcopenic participants or poorer QoL for sarcopenic patients, but generally only for specific QoL domains [8]. At the same time, generic questionnaires are used for measuring QoL in sarcopenia and this can further limit the literature available about the potential associations between sarcopenia and QoL [8]. Importantly, only two of the included studies were longitudinal in nature. In one study consisting of 670 older adults from Taiwan, it was observed that sarcopenia at baseline was associated with worse QoL scores at 4-year follow-up [9]. The other longitudinal study consisted of a sample of 230 patients listed for liver transplant and found no association between baseline sarcopenia and QoL at follow-up [10]. Other more recent studies, and thus not include in the review, have investigated the sarcopenia/QoL relationship in those with specific medical conditions e.g. dementia [11] or cancer [12]. It is clear that further research of a longitudinal nature is needed, with a longer follow-up, in large representative samples of the general older adult population to further elucidate the association between sarcopenia and QoL.

Given this background, the aim of the present study was to examine the association between sarcopenia at baseline and QoL at 10-year follow-up in a large representative sample of older English adults.

\section{Methods}

\section{Study population}

This study is based on data from six waves (from Wave 2 to Wave 7) of the English Longitudinal Study of Ageing (ELSA), which is a prospective and nationally representative cohort of men and women living in England [13]. Wave 2 (baseline survey) was conducted in 2004-2005; the other waves were conducted every 2 years, until Wave 7 between 2014 and 2015. The ELSA study was approved by the London Multicentre Research Ethics Committee (MREC/01/2/91). Informed consent was obtained from all participants.

\section{Sarcopenia (independent variable)}

Following the criteria of the revised European consensus on the definition and diagnosis of sarcopenia [14], sarcopenia was defined as weak handgrip strength and having low skeletal muscle mass (SMM), as reflected by lower skeletal mass index (SMI). Briefly, SMM was calculated based on the equation proposed by Lee and colleagues[15]:

SMM $=0.244 *$ weight $+7.8 *$ height $+6.6 *$ sex $-0.098 *$ age + race -3.3

( where female $=0$ and male $=1$; race $=0$ [White and Hispanic], race $=1.4$ [Black] and race $=-1.2$ [Asian] $)$.

SMM was further divided by body mass index (BMI) based on weight and height measured by a trained nurse, to create a SMI [16]. Low SMM was defined as the lowest quartile of the SMI based, on sex-stratified values [17]. Weak handgrip strength was defined as $<27 \mathrm{~kg}$ for men and $<16 \mathrm{~kg}$ for women using the average value of three handgrip measurements of the dominant hand [14]. Grip strength in kilograms was measured by using a Smedley dynamometer (TTM; Tokyo, Japan), with the upper arm being held against the trunk and the elbow in a 90-degree flexion [13]. We also investigated the onset of sarcopenia during the follow-up waves.

\section{Outcomes: quality of life}

The QoL measure used in the ELSA is CASP (control, autonomy, self-realisation and pleasure)-19 [18]. It is a selfcompletion questionnaire and spans four derived dimensions based on Likert scaled items. CASP-19 has an overall summary measure on a 0-57 scale, with higher scores corresponding to greater well-being [18]. CASP-19 was evaluated in each wave included in this analysis, from wave 2 to 7 . 
Poor QoL was defined, arbitrarily, as values falling in the lowest quartile values of CASP-19 in each wave.

\section{Covariates}

The selection of covariates was based on their previously reported associations with the exposure (sarcopenia) and outcome (poor QoL), and included the following: age; sex; years of education (considered as continuous variable); ethnicity (whites vs. non-whites); marital status (married vs. other status); smoking status (ever vs. never); physical activity level (high vs. moderate/low/sedentary): in the ELSA study, for assessing physical activity level, three questions were made regarding vigorous, moderate, mild activity in the previous twelve months. To assist in answering the questions, prompt cards with examples of activities categorised by intensity [19]; the presence of depressive symptoms assessed with the Center for Epidemiologic Studies Depression Scale (CES-D) [20]; the presence of multimorbidity was defined as $\geq 2$ chronic conditions [21], among all the medical conditions assessed at wave 2. All these parameters were assessed at baseline.

\section{Statistical analyses}

The data were weighted using the person-level longitudinal weight, core sample, wave 2 (http://www.ifs.org.uk/ELSA). The analysis was restricted to those aged $\geq 60$ years at baseline as sarcopenia is an age-related condition.

Continuous variables were analyzed in terms of distribution using the Kolmogorov-Smirnov test, using the Levene's test to test the homoscedasticity of variances. Means and standard deviations (SD) were used to describe quantitative measures, while percentages and counts were used for categorical variables. Characteristics of the study participants at baseline (wave 2) were compared according to the presence of sarcopenia at wave 2 with the use of Chi-squared or Fisher exact tests for categorical variables, and independent $t$ test for continuous variables.

The association between sarcopenia at baseline and poor quality of life during follow-up was assessed using univariable and multivariable logistic regression analysis and reported as odds ratios (OR) and $95 \%$ confidence intervals (95\% CI). Participants falling in the lowest quartile of CASP-19 at the baseline evaluation were removed from this analysis. Moreover, the association between sarcopenia at the baseline and the changes of CASP-19 during followup was evaluated using a generalized linear model with repeated measures, adjusting for the potential confounders mentioned in the covariates paragraph, including CASP-19 values at baseline. For missing data during follow-up regarding CASP-19, a multiple imputation method was used, with a maximum of 20 interactions [22]. For further minimizing the possible bias, we used a case-control match (1:10) using similar values of CASP-19 at wave 2 between people with and without sarcopenia.

All statistical tests were two-tailed, and a $p$ value $<0.05$ was considered to be statistically significant. All analyses were performed using SPSS 21.0 version software.

\section{Results}

A total of 9432 participants were included in the second wave (baseline) of the ELSA. In the present analyses 3186 participants were excluded owing to having an
Fig. 1 Flowchart of the selection of participants

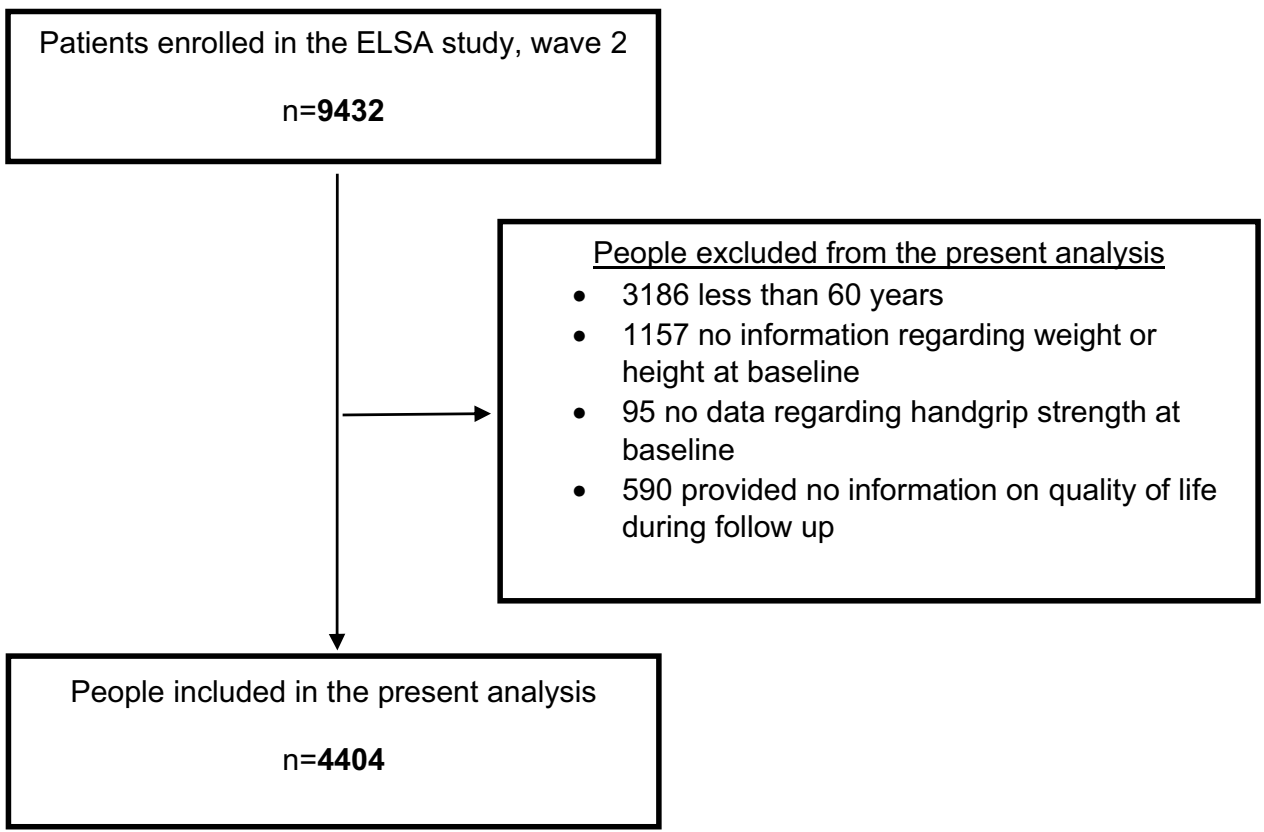


Table 1 Baseline characteristics by presence or absence of sarcopenia

\begin{tabular}{lllc}
\hline Parameter & Sarcopenia $(n=376)$ & $\begin{array}{l}\text { No sarcopenia } \\
(n=4028)\end{array}$ & $p$ value \\
\hline Age (mean, SD) & $79.7(7.4)$ & $69.8(7.0)$ & $<0.0001$ \\
Female gender $(n, \%)$ & $147(39.1)$ & $1829(45.4)$ & 0.02 \\
Years of education (mean, SD) & $3.4(5.8)$ & $6.7(6.9)$ & $<0.0001$ \\
Whites $(n, \%)$ & $363(96.5)$ & $3977(98.7)$ & 0.002 \\
Married $(n, \%)$ & $153(40.7)$ & $2564(65.9)$ & $<0.0001$ \\
Ever smoked $(n, \%)$ & $236(62.8)$ & $2530(62.8)$ & 1.00 \\
High physical activity level $(n, \%)$ & $22(5.9)$ & $726(18.09$ & $<0.0001$ \\
CASP-19 (mean, SD) & $31.3(17.3)$ & $35.8(11.7)$ & $<0.0001$ \\
CES-D (mean, SD) & $2.22(1.99)$ & $1.43(1.83)$ & $<0.0001$ \\
Multimorbidity (n, \%) & $309(82.2)$ & $2568(63.8)$ & $<0.0001$ \\
\hline
\end{tabular}

$S D$ standard deviation, $C A S P-19$ control, autonomy, self-realisation and pleasure-19, $C E S$ - $D$ center for epidemiologic studies depression scale age $<60$ years, 1157 owing to missing wave 2 data on body composition, 95 owing to missing wave 2 data on handgrip strength, and 590 owing to missing data on QoL at followup. The present analytic study population included 4044 participants (Fig. 1, unweighted data).

Participants mean age was $70.7 \pm$ SD 7.6 years (range 60-90) and $55.1 \%$ were female. The baseline prevalence of sarcopenia was $8.5 \%$. Table 1 shows the main descriptive characteristics of the participants included, according to the presence or absence of sarcopenia at wave 2 . Those who were sarcopenic at baseline $(n=376)$ were significantly older, more likely to be male, not married, and had a lower level of education compared to those without sarcopenia $(n=4028)$ (all $=p<0.0001)$. People with sarcopenia were less physically active, more depressed, and had worse CASP-19 scores, at baseline $(p<0.0001)$ (Table 1).

Table 2 shows the association between sarcopenia at baseline and the onset of poor QoL. In the univariable analysis, sarcopenia at baseline was associated with a higher incidence of poor QoL $(\mathrm{OR}=10.14 ; 95 \%$ CI 6.29-16.35; $p<0.0001)$ : this association remained statistically significant after adjusting for potentially important confounding variables $(\mathrm{OR}=5.82 ; 95 \% \mathrm{CI}$ 3.45-9.82; $p<0.0001$ ) (Table 2).

Figure 2 shows the association between those with sarcopenia $(n=269)$ and thosewithout sarcopenia at baseline $(n=2690)$ in terms of QoL during follow-up, after baseline matching of CASP-19 scores. After 10 years of follow-up, people with sarcopenia reported significantly lower values in CASP-19 (mean difference $=-3.94 ; 95 \%$ CI -4.77 to $-3.10 ; p<0.0001)$, after adjusting for potentially important confounding variables.

\section{Discussion}

In the present large representative sample of older adults with 10 years of follow-up, it was observed that the presence of sarcopenia at baseline was significantly associated with lower scores of QoL at follow-up compared to those who were not sarcopenic.

The present findings support and add to the only other longitudinal analyses carried out in a small sample $(n=670)$
Table 2 Association between sarcopenia at the baseline and incident poor quality of life (weighted data)

\begin{tabular}{lllll}
\hline & $\begin{array}{l}\text { Univariable model (OR, } \\
95 \% \text { CI })\end{array}$ & $p$-value & $\begin{array}{l}\text { Fully-adjusted } \text { model }^{\mathrm{a}} \\
(\mathrm{OR}, 95 \% \mathrm{CI})\end{array}$ & $p$-value \\
\hline No sarcopenia & Reference & - & Reference & - \\
Sarcopenia & $10.14(6.29-16.35)$ & $<0.0001$ & $5.82(3.45-9.82)$ & $<0.0001$ \\
\hline
\end{tabular}

Data are reported as odds ratios (ORs) with their $95 \%$ confidence intervals (CIs)

Incident poor quality of life was defined as the lowest quartile in control, autonomy, self-realisation and pleasure-19 values, specific for each wave

âFully-adjusted model included: age (as continuous variable); gender; years of education (as continuous variable); ethnicity (whites vs. non-whites); marital status (married vs. other status); smoking status (ever vs. never); Center for Epidemiologic Studies Depression Scale (as continuous variable); physical activity level (high vs. others); presence of multimorbidity (yes vs. no); control, autonomy, self-realisation and pleasure-19 values at baseline; changes in sarcopenia status during follow-up period 


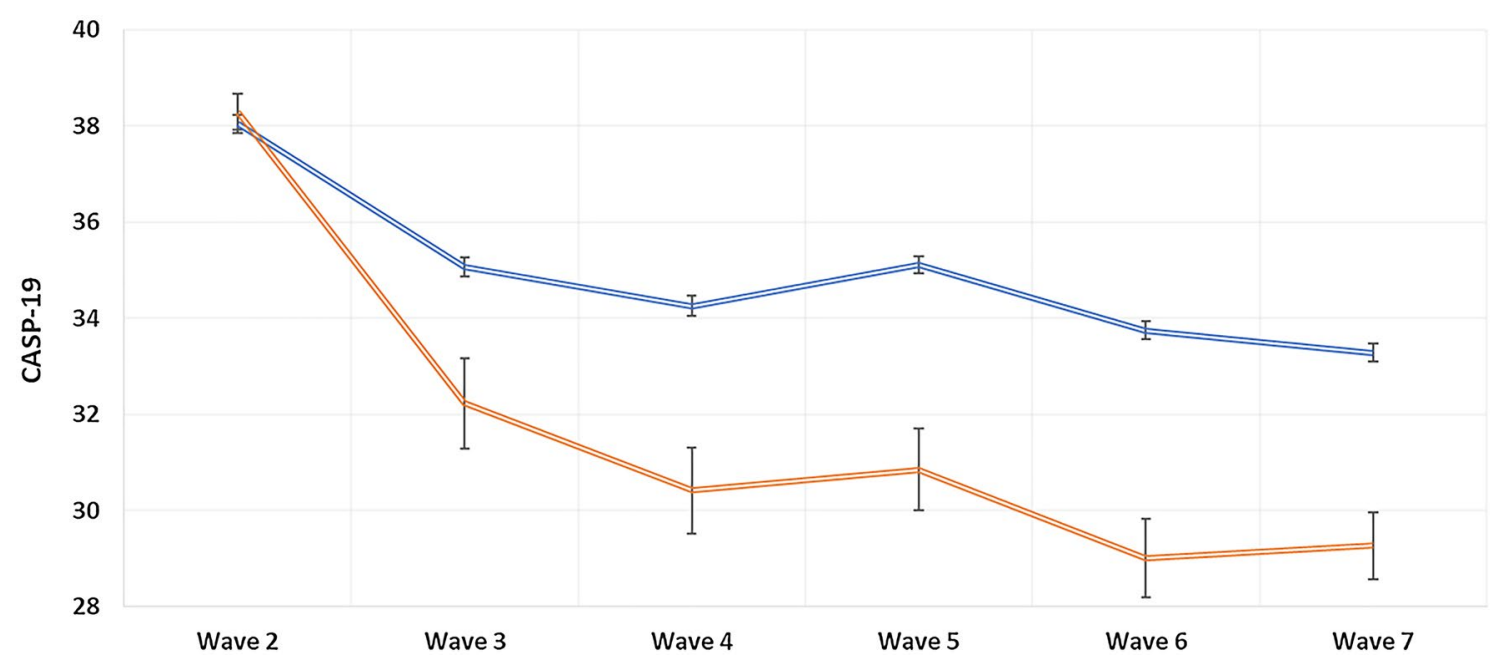

Fig. 2 Changes of quality of life, during follow-up, by presence of sarcopenia or not at baseline (weighted data). Data are reported as mean and standard errors by presence of sarcopenia (red line) and absence of this condition (blue line), matching people with and without sarcopenia for CASP-19 values at wave 2. Analyses were adjusted for age (as continuous variable); gender; years of education (as con-

of Taiwanese older adults and observed that sarcopenia at baseline was associated with worse QoL scores at 4-year follow-up [9]. It adds to this study through demonstrating that such an association holds in a large sample (4044) of older English adults with 10 years of follow-up. There are several plausible pathways that likely explain the association between sarcopenia and worse QoL scores. First, sarcopenia is associated with a greater risk of functional impairment and disability, which may limit one's ability to carry out certain functions in their daily lives [23] potentially impacting their QoL [24]. Second, sarcopenia is associated with higher risk of falling [25] and, therefore, may experience fractures owing to such falls or avoidance of specific behaviors owing to fear of falling, both of which may limit activities of daily life and thus reduce QoL [25]. Third, sarcopenia has been observed to be associated with multiple mental health complications [26] and mental health complications are indeed associated with a worsening of QoL [27]. Fourth, sarcopenia has been found to reduce sleep quality [28] potentially owing to a low level of irisin [29]. Poor sleep likely worsens QoL owing to excessive daytime sleepiness and lack of energy.

The findings from the present study and the only other longitudinal study in a sample of "healthy" older adults [9] suggest that it may be prudent for interventions to improve QoL to be targeted at those with sarcopenia. It may be beneficial to incorporate mind-body exercises into such interventions such as Tai-chi. Indeed, literature has shown that such exercises can significantly reduce sarcopenia symptoms [30] and improve QoL per se [31]. tinuous variable); ethnicity (whites vs. non-whites); marital status (married vs. other status); smoking status (ever vs. never); Center for Epidemiologic Studies Depression Scale (as continuous variable); physical activity level (high vs. others); presence of multimorbidity (yes vs. no)

The large representative sample and 10-year follow-up are strengths of the present study. However, findings must be interpreted in light of the studies limitations. Just one measure of muscle strength was employed to measure sarcopenia. Although a unified geriatric assessment tool has not as of yet been widely utilized to assess for sarcopenia, handgrip strength is indeed frequently implemented to measure muscle strength, which isa critical component of sarcopenia [14]. Handgrip strength is commonly employed in both research and clinical practice [32] and has been found to be an independent predictor of early mortality [33]. Next, the body composition estimate was calculated utilising a population equation and not direct assessment. In this sense, this equation did not consider some common anthropometric measures, such as hip circumference and the proposed equation was validated only in the USA, whilst no direct evaluation was made in Europe and particularly in the ELSA study [15]. Third, the CASP-19 has not been validated in sarcopenic people. Future similar research should seek to implement the SarQoL [34], which is the only validated QOL tool for use in people with sarcopenia, unfortunately, not available at the time of the baseline assessment of the ELSA study. Finally, it would be interesting to explore the possible association between sarcopenia and the altered dimensions of QoL, but this important information is unfortunately not available in CASP-19 scale [35].

In conclusion, in this large representative sample of older English adults, it was observed that sarcopenia at baseline was associated with worse scores of QoL at follow-up 
compared to those without sarcopenia at baseline. It may be prudent to target those with sarcopenia to improve QoL.

Acknowledgements The ELSA was developed by a team of researchers based at University College London, the National Centre for Social Research and the Institute for Fiscal Studies. The data were collected by the National Centre for Social Research. The funding was provided by the National Institute of Aging in the USA, and a consortium of UK government departments coordinated by the Office for National Statistics. The developers and funders of the ELSA and the UK Data Archive do not bear any responsibility for the analyses or interpretations presented here. J. W. is supported by the Centre for the Development and Evaluation of Complex Interventions for Public Health Improvement, a UKCRC Public Health Research: Centre of Excellence. Funding from the British Heart Foundation, Cancer Research UK, Economic and Social Research Council (ESRC RES-590-28-0005), Medical Research Council (MR/KO232331/1), the Welsh Assembly Government and the Wellcome Trust (WT087640MA), under the auspices of the UK Clinical Research Collaboration, and the contribution is gratefully acknowledged. M. K. is supported by the UK Medical Research Council (K013351), the Academy of Finland and the US National Institutes of Health (R01HL036310 and R01AG034454) and by a professorial fellowship from the Economic and Social Research Council. G. D. B. is a member of the University of Edinburgh Centre for Cognitive Ageing and Cognitive Epidemiology, part of the crosscouncil Lifelong Health and Wellbeing Initiative (G0700704/84698).

Funding None.

\section{Declarations}

\section{Conflict of interest None.}

Ethical approval The ELSA study was approved by the London Multicentre Research Ethics Committee (MREC/01/2/91).

Informed consent Informed consent was obtained from all participants in written form.

Open Access This article is licensed under a Creative Commons Attribution 4.0 International License, which permits use, sharing, adaptation, distribution and reproduction in any medium or format, as long as you give appropriate credit to the original author(s) and the source, provide a link to the Creative Commons licence, and indicate if changes were made. The images or other third party material in this article are included in the article's Creative Commons licence, unless indicated otherwise in a credit line to the material. If material is not included in the article's Creative Commons licence and your intended use is not permitted by statutory regulation or exceeds the permitted use, you will need to obtain permission directly from the copyright holder. To view a copy of this licence, visit http://creativecommons.org/licenses/by/4.0/.

\section{References}

1. Group W (1993) Study protocol for the World Health Organization project to develop a Quality of Life assessment instrument (WHOQOL). Qual Life Res 2:153-159

2. Group W (1995) The World Health Organization quality of life assessment (WHOQOL): position paper from the World Health Organization. Soc Sci Med 41:1403-1409
3. Phyo AZZ, Freak-Poli R, Craig H, Gasevic D, Stocks NP, Gonzalez-Chica DA, Ryan J (2020) Quality of life and mortality in the general population: a systematic review and meta-analysis. BMC Public Health 20:1-20

4. Otero-Rodríguez A, León-Muñoz LM, Balboa-Castillo T, Banegas JR, Rodríguez-Artalejo F, Guallar-Castillón P (2010) Change in health-related quality of life as a predictor of mortality in the older adults. Qual Life Res 19:15-23

5. Walston JD (2012) Sarcopenia in older adults. Curr Opin Rheumatol 24:623-627

6. Anker SD, Morley JE, von Haehling S (2016) Welcome to the ICD-10 code for sarcopenia. J Cachexia Sarcopenia Muscle $7: 512-514$

7. Woo J (2017) Sarcopenia. Clin Geriatr Med 33:305-314

8. Beaudart $\mathrm{C}$, Reginster J-Y, Geerinck $\mathrm{A}$, Locquet $\mathrm{M}$, Bruyère $\mathrm{O}$ (2017) Current review of the SarQoL®: a health-related quality of life questionnaire specific to sarcopenia. Expert Rev Pharmacoecon Outcomes Res 17:335-341

9. Wu T-Y, Liaw C-K, Chen F-C, Kuo K-L, Chie W-C, Yang R-S (2016) Sarcopenia screened with SARC-F questionnaire is associated with quality of life and 4-year mortality. J Am Med Dir Assoc 17:1129-1135

10. Yadav A, Chang YH, Carpenter S, Silva AC, Rakela J, Aqel BA, Byrne TJ, Douglas DD, Vargas HE, Carey EJ (2015) Relationship between sarcopenia, six-minute walk distance and health-related quality of life in liver transplant candidates. Clin Transplant 29:134-141

11. Umegaki H, Bonfiglio V, Komiya H, Watanabe K, Kuzuya M (2020) Association between sarcopenia and quality of life in patients with early dementia and mild cognitive impairment. J Alzheimers Dis 76:435-442

12. Nipp RD, Fuchs G, El-Jawahri A, Mario J, Troschel FM, Greer JA, Gallagher ER, Jackson VA, Kambadakone A, Hong TS (2018) Sarcopenia is associated with quality of life and depression in patients with advanced cancer. Oncologist 23:97

13. Steptoe A, Breeze E, Banks J, Nazroo J (2013) Cohort profile: the English longitudinal study of ageing. Int J Epidemiol 42:1640-1648

14. Cruz-Jentoft AJ, Bahat G, Bauer J et al (2019) Sarcopenia: revised European consensus on definition and diagnosis. Age Ageing 48:16-31

15. Lee RC, Wang Z, Heo M, Ross R, Janssen I, Heymsfield SB (2000) Total-body skeletal muscle mass: development and cross-validation of anthropometric prediction models. Am J Clin Nutr 72:796-803

16. Studenski SA, Peters KW, Alley DE et al (2014) The FNIH sarcopenia project: rationale, study description, conference recommendations, and final estimates. J Gerontol A Biol Sci Med Sci 69:547-558

17. Tyrovolas S, Koyanagi A, Olaya B, Ayuso-Mateos JL, Miret M, Chatterji S, Tobiasz-Adamczyk B, Koskinen S, Leonardi M, Haro JM (2016) Factors associated with skeletal muscle mass, sarcopenia, and sarcopenic obesity in older adults: a multicontinent study. J Cachexia Sarcopenia Muscle 7:312-321

18. Howel D (2012) Interpreting and evaluating the CASP-19 quality of life measure in older people. Age Ageing 41:612-617

19. McMullan II, Bunting BP, McDonough SM, Tully MA, Casson K (2020) The association between light intensity physical activity with gait speed in older adults ( $\geq 50$ years). A longitudinal analysis using the English Longitudinal Study of Ageing (ELSA). Aging Clin Exp Res 32:2279-2285

20. Eaton WW, Smith C, Ybarra M, Muntaner C, Tien A (2004) Center for Epidemiologic Studies Depression Scale: review and revision (CESD and CESD-R). 
21. Garin N, Koyanagi A, Chatterji S et al (2016) Global multimorbidity patterns: a cross-sectional, population-based, multicountry study. J Gerontol A Biol Sci Med Sci 71:205-214

22. Royston P (2004) Multiple imputation of missing values. Stand Genomic Sci 4:227-241

23. Janssen I, Heymsfield SB, Ross R (2002) Low relative skeletal muscle mass (sarcopenia) in older persons is associated with functional impairment and physical disability. J Am Geriatr Soc 50:889-896

24. Sahoo P, Sethy RR, Ram D (2017) Functional impairment and quality of life in patients with obsessive compulsive disorder. Indian J Psychol Med 39:760-765

25. Yeung SS, Reijnierse EM, Pham VK, Trappenburg MC, Lim WK, Meskers CG, Maier AB (2019) Sarcopenia and its association with falls and fractures in older adults: a systematic review and meta-analysis. J Cachexia Sarcopenia Muscle 10:485-500

26. Chang K-V, Hsu T-H, Wu W-T, Huang K-C, Han D-S (2017) Is sarcopenia associated with depression? A systematic review and meta-analysis of observational studies. Age Ageing 46:738-746

27. Brenes GA (2007) Anxiety, depression, and quality of life in primary care patients. Primary Care Compan J Clin Psychiatry 9:437

28. Nagaura Y, Kondo H, Nagayoshi M, Maeda T (2020) Sarcopenia is associated with insomnia in Japanese older adults: a cross-sectional study of data from the Nagasaki Islands study. BMC Geriatr 20:1-8

29. Park H-S, Kim HC, Zhang D, Yeom H, Lim S-K (2019) The novel myokine irisin: clinical implications and potential role as a biomarker for sarcopenia in postmenopausal women. Endocrine 64:341-348
30. Morawin B, Tylutka A, Chmielowiec J, Zembron-Lacny A (2021) Circulating mediators of apoptosis and inflammation in aging; physical exercise intervention. Int J Environ Res Public Health 18:3165

31. Wang D, Wang P, Lan K, Zhang Y, Pan Y (2020) Effectiveness of Tai chi exercise on overall quality of life and its physical and psychological components among older adults: a systematic review and meta-analysis. Braz J Med Biol Res 53:3

32. Roberts HC, Denison HJ, Martin HJ, Patel HP, Syddall H, Cooper C, Sayer AA (2011) A review of the measurement of grip strength in clinical and epidemiological studies: towards a standardised approach. Age Ageing 40:423-429

33. Wu Y, Wang W, Liu T, Zhang D (2017) Association of grip strength with risk of all-cause mortality, cardiovascular diseases, and cancer in community-dwelling populations: a meta-analysis of prospective cohort studies. J Am Medical Direct Assoc 18:551

34. Beaudart C, Biver E, Reginster JY, Rizzoli R, Rolland Y, Bautmans I, Petermans J, Gillain S, Buckinx F, Dardenne N (2017) Validation of the SarQoL®, a specific health-related quality of life questionnaire for Sarcopenia. J Cachexia Sarcopenia Muscle 8:238-244

35. Veronese N, Smith L, Pizzol D, Soysal P, Maggi S, Ilie P-C, Dominguez LJ, Barbagallo M (2022) Urinary incontinence and quality of life: a longitudinal analysis from the English Longitudinal Study of Ageing. Maturitas

Publisher's Note Springer Nature remains neutral with regard to jurisdictional claims in published maps and institutional affiliations. 